\title{
DETERMINATION OF THE DEPTH OF CLOSED BLIND CRACKS IN NON-METAL CHECK SAMPLES AND TEST PANELS FOR PENETRANT TESTING
}

\author{
Nikolay Kalinichenko ${ }^{1}$, Aleksey Kalinichenko ${ }^{1}$, Irina Lobanova ${ }^{1 *}$, Anna Zaitseva ${ }^{1}$, \\ Egor Loboda ${ }^{2}$ \\ ${ }^{1}$ National Research Tomsk Polytechnic University, 634050, Tomsk, Russia \\ ${ }^{2}$ National Research Tomsk State University, 634050, Tomsk, Russia
}

\begin{abstract}
Check samples and test panels are used to quality assessment and comparing sensitivity of sets of penetrant material. Authors made research in field of manufacturing non-metal test-objects with specified parameters, such as length a width. The paper considers a technique to determine the depth of blind closed cracks in check samples and non-metal test panels for penetrant inspection with a special tool to measure the depth of the undercut depth or a gauge indicator on the outboard support.
\end{abstract}

\section{Introduction}

A new version of the International Standard for non-destructive penetrant testing was approved in the late 2006s. The new standard allows the use of different check samples which satisfy specified requirements. However, the most common check samples which meet the International standard Non-destructive testing. Penetrant testing. Part 3: Reference test blocks (EN ISO 3452-3) standard in the European Union are expensive, and in many cases impracticable because they lead to high values of sensitivity levels $[1,2]$.

Among check samples used abroad, the one to be considered is a set of four plates with nickel-chromium coating with a thickness of 10, 20, 30 and $50 \mu \mathrm{m}$ (type 1 sample) [3].

The accuracy of the coating thickness uniformity is about $10 \%$. Transverse cracks on each panel are produced by longitudinal stretching of the panel. The plates with a coating thickness of 10,20 and $30 \mu \mathrm{m}$ are used to determine the sensitivity level of the fluorescent penetrant systems. The sensitivity of the systems based on color-contrast penetrants is determined by the 30 and $50 \mu \mathrm{m}$ plates.

The check samples corresponding to EN ISO 3452-3 [3] used to determine the level of sensitivity of flaw detection sets may result in high values. This is due to the U-shaped form of cracks in these samples which have entry to the side of the open cracks. When the penetrant is applied to these samples, the air in the crack cavity is squeezed, and the liquid penetrant easily enters the cavity. Therefore, in some cases, even low sensitivity penetrant

*Corresponding author: konarevai007@tpu.ru 
can provide sufficiently bright contrasting indicator traces referred to a higher sensitivity class.

Penetration of the penetrant in the cavity of the cracks formed in the central part on the check sample surface, i.e. closed cracks, proceeds in a different manner. In this case, the air closed up in the defect cavity prevents its rapid completion by the penetrant, and this is a sufficiently easier way to identify the penetration characteristics during a series of operations [1].

Typically, the passports of check samples for penetrant testing made of metal through nitriding [4-7] with the following exposure to loading indicate that a crack depth is equal to the thickness of the nitrided layer with no numerical values provided. In recent years, the manufacturers of check samples do not report any data on the crack depth since the measurement of the crack depth in the range of tens to hundreds of micrometers is problematic.

The technique that employs non-metal check samples and test panels to eliminate this drawback is considered below.

\section{Technology of non-metal test sample manufacturing}

The technology of non-metal test sample manufacturing reported in [8] allows manufacturing defects of the designed parameters: length, depth and disclosure, and variation of these parameters.

When implementing an open crack which extends from one side edge to the other side edge of the test sample, this technology allows measurement of the crack depth on both sample edges and calculation of an average depth value.

The variability of the crack depth during manufacturing exhibits the following advantages of the proposed technology. As an example, after manufacturing of the check sample using a $1 \mathrm{~mm}$ foil strip, the crack depth can be reduced in further treatment to virtually any desired value $(10-500) \mu \mathrm{m}$, which will determine the defect depth after etching.

The studies that address the problem of manufacturing non-metal check samples allow provide with the technology of manufacturing test panel with both open and closed blind cracks for penetrant testing. However, several cracks of different sensitivity classes may be implemented on the test surface [9].

As mentioned above, the problem to be faced when manufacturing check samples and test panels with closed blind cracks is measuring the crack depths.

Consider one of the following techniques of manufacturing this type of cracks in nonmetal test panels with the ability to control the crack depth. A non-metal (based on epoxy adhesive) bar of the size smaller than that of the future test panel is prepared. Then, metal strips of equal length and width are cut. In this case, the strip workpieces were made of the following materials:

- mica plate with a deposited layer of silver;

- silver leaf;

- metal foil.

The problem of implementation of test panels, particularly of sensitivity class I, is the absence of foil of required thickness (less than $1 \mu \mathrm{m}$ ). However, mica plates coated with the silver layer (approximately 1 micron) can be used instead.

The strips are adhered in pairs (of equal thickness) opposite to each other on the sides of the bar in its lower (bottom) part with epoxy adhesive (Figure 1). 


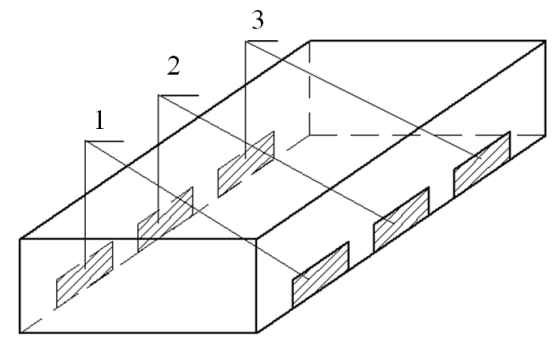

Figure 1. Rectangular non-metal bar with fixed strips 1-3.

Then, the bar is placed in the pre-prepared larger mold that will determine the size of the test panel. The mold is filled with epoxy adhesive up to the desired level. Within the first minutes of its solidification, an ultrasonic bath is used to reduce the amount of bubbles.

After epoxy solidification, the workpiece is removed from the mold, grinded to the required parameters of cracks (especially depth) and polished. In this case, the bottom surface of the panel is its test surface.

The technique to control the crack depth in the investigated test panel is proposed below. Several $2-3 \mathrm{~mm}$ diameter holes are to be drilled in the workpiece test (bottom) surface, as shown in Figure 2.

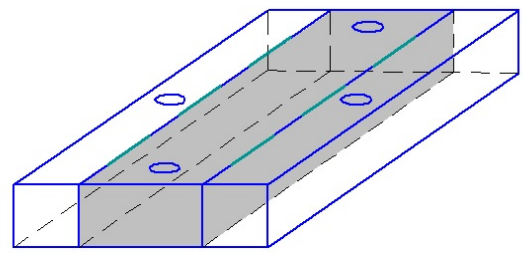

Figure 2. Rectangular non-metal test panel with drilled holes.

The hole depth is equal to the width of the adhered metal strips. During treatment, the hole depth and thus the remaining strip width may be controlled using a special tool to measure the undercut depth [10], i.e. the dial depth gauge with outboard support (Figure 3, $a, b$ ). These tools employ an indicating gage with the measurement limit of $0-$ $10 \mathrm{~mm}$ and resolution of $10 \mu \mathrm{m}$ as a reading device. You can also use a high-precision digital vernier caliper designed for welds (Figure 3,c).

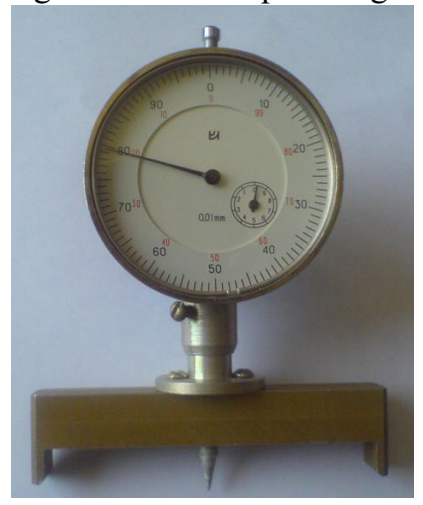

a

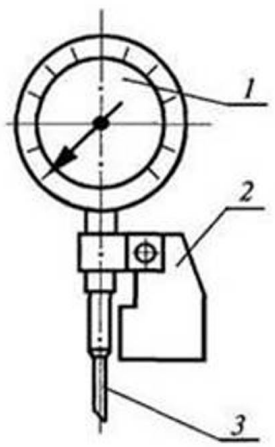

b

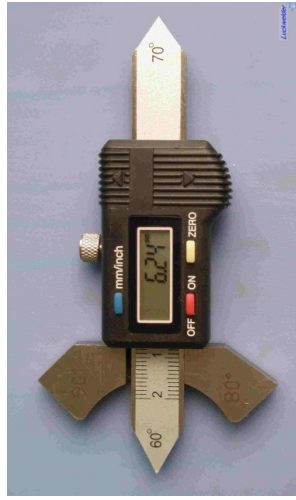

c

Figure 3. Dial depth gauge with outboard support $(\mathrm{a}, \mathrm{b}, \mathrm{c})$; tool to measure the depth of undercuts: indicator " $0-10$ " with a rotating scale (1); 2 - support bracket; 3 - measuring pin. 
Excessive grinding of the sample material during treatment leads to unevenness of both the strip width and the crack depth. The deviation of the crack depth unevenness (defect) flatness can be significantly reduced by using the technique of non-metal check sample manufacturing considered further on. In addition to compliance with the standards recommended for the metal section manufacturing [11], metal strips should be placed at the workpiece edge maximum distance. Since the strips are located in the center of the workpiece at a considerable distance from the workpiece edge, the width of the remaining metal strips after treatment is almost equal. This makes the crack depths virtually equal after etching of the remaining strips.

When the width of metal strips reduces as a result of the workpiece test surface treatment, the hole depth decreases. The width of the metal strips is controlled by measuring the hole depth using a dial depth gauge or the tool to measure the depth of undercuts. The holes in different areas of the test surface makes possible to control the width of metal strips to make it uniform. In the end of treatment, the required width is achieved in metal strips, which ultimately determine the crack depth in the test panel. The resulting treatment of the bar test surface must be performed with the abrasive material of a minimal grit to provide a minimal test surface when using a test panel for further work.

After that, the groove is cut in the test surface to divide the test panel into two symmetrical parts (Figure 4). With this, traces of holes in the central part of the panel are eliminated. The remaining holes will not interfere with the analysis of the test results, as the holes are wide and shallow and do not add any indicator traces.

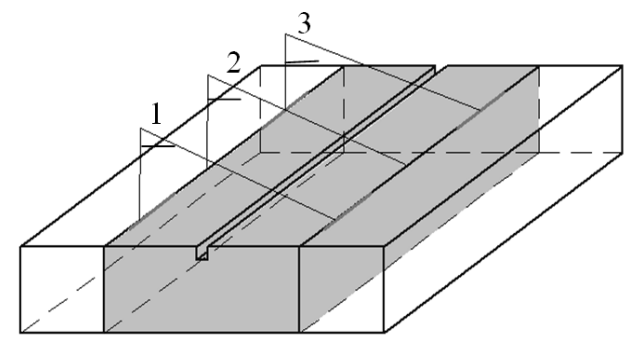

Figure 4. Test panel according to sensitivity classes I-III: 1 - crack of sensitivity class III; 2 - crack of sensitivity class II; 3 - crack of sensitivity class I.

The remains of the metal foil are etched with ferric chloride solution. The obtained cracks are tested for the opening width.

Consider another technique to determine the depth of cracks. Since the dividing groove implementation is required for manufacturing of these test panels, the groove can be used to control the crack depth.

In doing so, the dividing groove of uniform depth, which is not less than the width of metal strips, is cut before the treatment of the bottom surface of the sample. Further treatment of the sample surface enables the control of the groove depth along its entire length, and therefore the width of strips to obtain the required values. In this embodiment, no control holes are required.

Consider another technique for manufacturing the test panel according to different sensitivity classes with the ability to control the depth of blind closed cracks. In our opinion, it is the most manufacturable one. To implement the technique, we need to:

- make the mold of the size equal to that of the future test panel;

- prepare metal strips to implement the cracks with the required parameters;

- adhere the pairs of metal strips to the bottom surface opposite to each other;

- fill the mold with epoxy adhesive; 
- cut a groove of the depth more than the strip width $(2-3 \mathrm{~mm})$ in the center of the bar bottom surface between the rows of the strips after epoxy curing;

- treat the bottom surface unless the required strip width (the depth of future cracks) is achieved;

- etch the remaining metal strips;

- control the parameters of the obtained dimensions of the blind closed cracks.

The parameters of the test panel implemented according to the technique proposed are as follows:

- opening width of cracks: No. $1-0.9 \mu \mathrm{m}$, No. $2-2 \mu \mathrm{m}$, No. $3-15 \mu \mathrm{m}$;

- crack length: $25 \mathrm{~mm}$;

- crack depth: $100 \mu \mathrm{m}$.

\section{Conclusion}

The techniques proposed to control the depth of blind cracks in check samples and nonmetal test panels enable a high accuracy in determination of their depth.

Manufacturing of samples with a wide range of crack depths allows for various practical studies on hydrodynamics of the defect backfill and development, and on the nature of the impact of external factors (temperature, humidity, etc.) on the defect parameters.

\section{References}

[1] N.P. Migun, N.B. Gnusin, Heat exposure at the capillary non-destructive testing (Nauka, Minsk, 2011)

[2] Zh. Zhang, H. Feng, W. Zhao, MATEC Web of Conferences 40, 04001 (2016) doi: $10.1051 /$ matecconf $/ 20164004001$

[3] ISO 3452-3-1998 International standard Non-destructive testing. Penetrant testing. Part 3: Reference test blocks (ITD)

[4] J.M. William, Test piece for inspection penetrant performance assessment and comparison US Patent 6729175 B2 (2004)

[5] F. J. Vicki, S. Shimizu, A Surface penetrant inspection test piece having varying thickness plating US Patent 4610157 (1986)

[6] W. J. Martin, Test piece for inspection penetrant performance assessment and comparison Patent US 6311538 B1 (2001)

[7] P. Dunnwald, Non-destructive liquid penetrant inspection process integrity verification test panel Patent WO 2011097146 A1 (2011)

[8] N.P. Kalinichenko, A.N. Kalinichenko, I.S. Lobanova, A.Y. Popova, S.S. Borisov, Meas. Tech. 57, 484 (2014) doi: 10.1007/s11018-014-0485-1

[9] N.P. Kalinichenko, A.N. Kalinichenko, I.S. Lobanova, A.A. Zaitseva, E.L. Loboda, J. Phys.: Conf. Ser. 671, 012033 (2016) doi: 10.1088/1742-6596/671/1/012033

[10] RD 03-606-03, Instruction on visual testing (Gosgortechnadzor of Russia)

[11] Yu.P. Egorov, Collection of guidelines for laboratory works on the course "Materials" (Tomsk Polytechnic University, Tomsk, 2014) 